\title{
Treosulfan Plus Fludarabine - Encouraging Conditioning for Allogeneic Hematopoietic Stem Cell Transplantation in Multiple Myeloma
}

\author{
Nahi $\mathrm{H}^{* 1}$, Afram $\mathrm{G}^{1}$, Liwing $\mathrm{J}^{1}$, Heeg $\mathrm{B}^{2}$, Mattsson $\mathrm{J}^{3}$, Katarina $\mathrm{LB}^{3}$, Björkstrand $\mathrm{B}^{1}$, Gahrton $\mathrm{G}^{1}$ and \\ Ljungman $\mathrm{P}^{3}$ \\ ${ }^{1}$ Department of Medicine, Karolinska Institute, Karolinska University Hospital, Stockholm, Sweden \\ ${ }^{2}$ Department of Epidemiology, University Medical Center Groningen, Groningen, Netherlands \\ ${ }^{3}$ Deptartment of Oncology and Pathology, Karolinska Institute and Center for allogeneic stem cell transplantation, \\ Karolinska University Hospital, Sweden
}

${ }^{*}$ Corresponding author: Nahi H, Department of Medicine, Karolinska Institutet, Karolinska University Hospital, Stockholm, Sweden, Tel: +46(0)858580000, E-mail: hareth.nahi@karolinska.se

Citation: Nahi H, Afram G, Liwing J, Heeg B, Mattsson J, et al. (2016) Treosulfan Plus Fludarabine Encouraging Conditioning for Allogeneic Hematopoietic Stem Cell Transplantation in Multiple Myeloma. J Cancer Sci Clin Oncol 3(2): 201. doi: 10.15744/2394-6520.3.201

Received Date: February 22, 2016 Accepted Date: September 06, 2016 Published Date: September 08, 2016

\begin{abstract}
Allogeneic transplantation (Allo-SCT) in multiple myeloma is controversial mainly due to the relatively high non-relapse mortality (NRM). The aim with this retrospective single-center study was to evaluate recent transplants particularly after conditioning with treosulfan plus fludarabine (TreoFlu). The 18 months overall survival (OS) for the patients allografted after and before year 2008 was $89 \%$ vs $66 \%$ respectively $(\mathrm{HR}=0.14(95 \% \mathrm{CI} 0.03-0.65, p=0.01)$ and for patients transplanted in $\geq \mathrm{VGPR}$ and $<\mathrm{VGPR} 72 \%$ vs $44 \%$ respectively $(\mathrm{HR}=0.49$ (95\% CI $0.17-1.39, p=0.18)$. Eleven of the 22 patients transplanted after 2008 received TreoFlu. Only one of these patients died of NRM. Thus, this single center study indicates improved survival following allogenic transplantation in multiple myeloma since 2008 perhaps in part due to encouraging use of TreoFlu for conditioning.
\end{abstract}

Allogeneic transplantation (Allo-SCT) for treatment of multiple myeloma (MM) is controversial mainly due to high transplant related mortality (or non relapse mortality - NRM) with myeloablative conditioning [1,2]. However, Allo-SCT is probably the only treatment that has resulted in cure of a small fraction of patients.

Treosulfan is a prodrug of a bifunctional alkylating agent which has both myelotoxic and immunosuppressive properties [3]. Recently a regimen of moderate dose treosulfan has been used in combination with fludarabine and ATG for treatment of malignant lymphoma and a small number of patients with multiple myeloma with low transplant related mortality [4,5]. In the present single center study we have used this combination in a small number of patients with encouraging results. In addition we have retrospectively compared the transplants performed at our center from 2008 with those performed before this time indicating an improvement in the transplant methodology with time.

Thirty-nine patients diagnosed with MM who had received an Allo-SCT between 2000 and 2015 at our center as part of $1^{\text {st }}$ line $(n=22,56 \%), 2^{\text {nd }}(n=13,34 \%)$ or later lines of treatment $(N=4,10 \%)$ were included in this retrospective study. Patients' characteristics are presented in Table 1. Analysis was made of two response groups: Very good partial remission or better ( $\geq$ VGPR) and less (worse) than very good partial remission ( $<\mathrm{VGPR}$ ) after transplant and of two "time of transplant" related groups (before and after 2008). Overall survival (OS) was calculated from the date of Allo-SCT until death (or censored at last follow-up). Nonrelapse mortality (NRM) was defined as any death beside deaths caused by MM. The median follow-up was 3.4 years for patients still alive and 2.1 years for the whole population. The local ethical committee, 2015/973-32, approved the study.

39 patients were included. 22 (patients received AlloSCT in $1^{\text {st }}$ remission, 13 patients in $2^{\text {nd }}$ remission and 4 patients beyond $2^{\text {nd }}$ remission. The conditioning regimens for AlloSCT were cyclophosphamide $60 \mathrm{mg} / \mathrm{kg}$ x $2+$ total-body irradiation (TBI), given in $64 \%$ of the cases, and in $30 \%$ of the patients fludarabin $30 \mathrm{mg} / \mathrm{m}^{2}$ x 5 and treosulfan $14 \mathrm{~g} / \mathrm{m}^{2} \times 3$ (Treo/Flu) was used. Two patients (5\%) received syngeneic transplantation 2001 and 2010, both still alive without signs of relapse. Of the 22 patients who were treated with AlloSCT from year 2008 and onwards, 50\% 11 received Treo/Flu as conditioning. All patients conditioned with TreoFlu were transplanted after 2008. Patients, who received stem cells from an unrelated donor (URD), also received ATG (Thymoglobulin, Genzyme Inc) $2.5 \mathrm{mg} / \mathrm{kg} /$ day, days $-3,-2,-1$. 


\begin{tabular}{|c|c|c|c|c|c|}
\hline & All & $<2008$ & $>2008$ & FluTreo & Other conditioning \\
\hline No, (\%) & $39(100)$ & $17(44)$ & $22(56)$ & $11(28)$ & $28(72)$ \\
\hline Age (range) & $48(24-60)$ & $52(35-60)$ & $46(24-60)$ & $51(34-60)$ & $48(24-60)$ \\
\hline Gender/female $\mathrm{Nr}(\%)$ & $13(33)$ & $5(29)$ & $8(36)$ & $5(45)$ & $8(29)$ \\
\hline $\begin{array}{l}\text { Unrelated donors } \\
\text { No, (\%) }\end{array}$ & $15(38)$ & $4(26)$ & $11(50)$ & $8(73)$ & $7(25)$ \\
\hline $\begin{array}{c}\text { Line of } 1^{\text {st }} \text { treatment } \\
2^{\text {nd }}>2^{\text {nd }}\end{array}$ & $\begin{array}{c}22(56) 13 \\
(33) 4 \\
(10)\end{array}$ & $\begin{array}{c}14(82) \\
1(6) \\
2(12)\end{array}$ & $\begin{array}{c}8(36) \\
12(55) \\
2(9)\end{array}$ & $\begin{array}{l}2(18) \\
7(64) \\
2(18)\end{array}$ & $\begin{array}{c}20(72) \\
6(21) \\
2(7)\end{array}$ \\
\hline $\begin{array}{l}\geq \text { VGPR before Allo- } \\
\text { SCT, } \operatorname{nr}(\%)\end{array}$ & $25(64)$ & $7(41)$ & $18(82)$ & $10(91)$ & $14(50)$ \\
\hline $\begin{array}{l}\geq \text { VGPR after Allo-SCT, } \\
\operatorname{nr}(\%)\end{array}$ & $30(77)$ & $9(53)$ & $21(95)$ & $10(91)$ & $20(71)$ \\
\hline $\begin{array}{c}\text { ISS at diagnosis, } \operatorname{nr}(\%) \\
\text { I } \\
\text { II } \\
\text { III }\end{array}$ & $\begin{array}{c}13(33) \\
20(51) \\
6(16)\end{array}$ & $\begin{array}{l}8(47) \\
7(41) \\
2(12)\end{array}$ & $\begin{array}{c}5(23) \\
13(59) \\
4(18)\end{array}$ & $\begin{array}{l}1(9) \\
8(73) \\
2(18)\end{array}$ & $\begin{array}{c}12(43) \\
12(43) \\
4(14)\end{array}$ \\
\hline $\begin{array}{c}\text { Poor prognosis Cytoge- } \\
\text { netics }\end{array}$ & $23(59)$ & $7(41)$ & $14(64)$ & $9(82)$ & $14(50)$ \\
\hline aGVHD, nr(\%) & $23(59)$ & $10(59)$ & $13(59)$ & $8(73)$ & $15(54)$ \\
\hline cGVHD, $\mathrm{nr}(\%)$ & $20(51)$ & $8(47)$ & $12(55)$ & $6(55)$ & $14(50)$ \\
\hline
\end{tabular}

Table 1: Patient characteristics and response to transplant

Sixteen patients developed acute graft-versus-host disease (aGVHD). Six patients developed grade I aGVHD, five patients with grade II and five patients with grade III. Eleven patients developed cGVHD, of which ten patients developed severe cGVHD as per NIH criteria and one patient was found to have moderate cGVHD. The most predominant organ manifestations were in the liver, the oral mucosa and the skin with an organ specific score of 1-3. Three patients developed pulmonary cGVHD with an organ specific score of 2 . One patient with pulmonary cGVHD died approximately 5 months after diagnosis due to progression of cGVHD and respiratory failure.

To assess impact of independent variables on PFS and OS univariate cox-regressions were conducted. The considered dependent variables were age, gender, whether patients were treated with TreoFlu after remission, response prior to treatment and response post treatment, match and whether patient underwent Allo-SCT prior or post 2008. Due to the low number of included patients, no multivariate analyses were conducted.

Age tended to be higher before 2008 and unrelated donors were more often used after 2008. More upfront transplants were performed before 2008. Poor cytogenetics tended to be more frequent after 2008 and particularly in the TreoFlu group The pretransplant overall response status was $\geq$ VGPR in $64 \%$ of the patients and tended to be worse in patients transplanted before 2008 as compared to those transplanted after 2008 ( $\geq$ VGPR before 2008: 41\% and after 82\%). Only 36\% of the patients were transplanted upfront ( $1^{\text {st }}$ line) after 2008 as compared to $82 \%$ before. Only $18 \%$ ( 2 patients) were treated upfront with TreoFlu while 9 patients were treated after progression (7 patient in $2^{\text {nd }}$ line and 2 patients in $3^{\text {rd }}$ line).

The 18 months OS for patients allografted after and before 2008 was $89 \%$ vs $66 \%$ respectively $(\mathrm{HR}=0.14(95 \% \mathrm{CI} 0.03-0.65, p=$ 0.01). The 18 months OS for patients treated with Treo/Flu was $89 \%$ compared to $69 \%$ with other conditioning regimens $(\mathrm{HR}=$ 0.14 (95\% CI $0.039-2.39, p=0.26$ ). (Table1 and Figure1). OS for the patients in $\geq$ VGPR and $<$ VGPR before transplant $80 \%$ and $43 \%$ respectively $(\mathrm{HR}=0.34(95 \% \mathrm{CI} 0.12-0.97, p=0.045)$.

The cumulative incidence of NRM was $12 \%$ in patients transplanted after 2008 (one due to respiratory insufficiency and one sudden death) and 22\% in the patients transplanted before 2008. In the TreoFlu treated patients, the cumulative incidence at 18 months was $9.1 \%$ (one patient). Ten patients relapsed or did not reach CR after Allo-SCT (>year 2008). Eight of these patients were treated with a lenalidomide-containing regimen. Due to different management of patients during the early and late phases of this study, an analysis of PFS was highly biased since the definitions and the indications differed a lot between patients transplanted in the early and late phases of the study period. In most patients anti-myeloma treatment (mainly with lenalidomide) was initiated before progression (25\% increase in M-protein or organ damage because of multiple myeloma), during the period DLI has been used as first line therapy in patients not in CR or progressing after SCT. After 2008, lenalidomide has been incorporated in the treatment strategy either after failure of DLI, alternating with DLI, or most recently as first line therapy sometimes followed by DLI. Because of the limitation of the material, no further assessment of the role of DLI was done. 


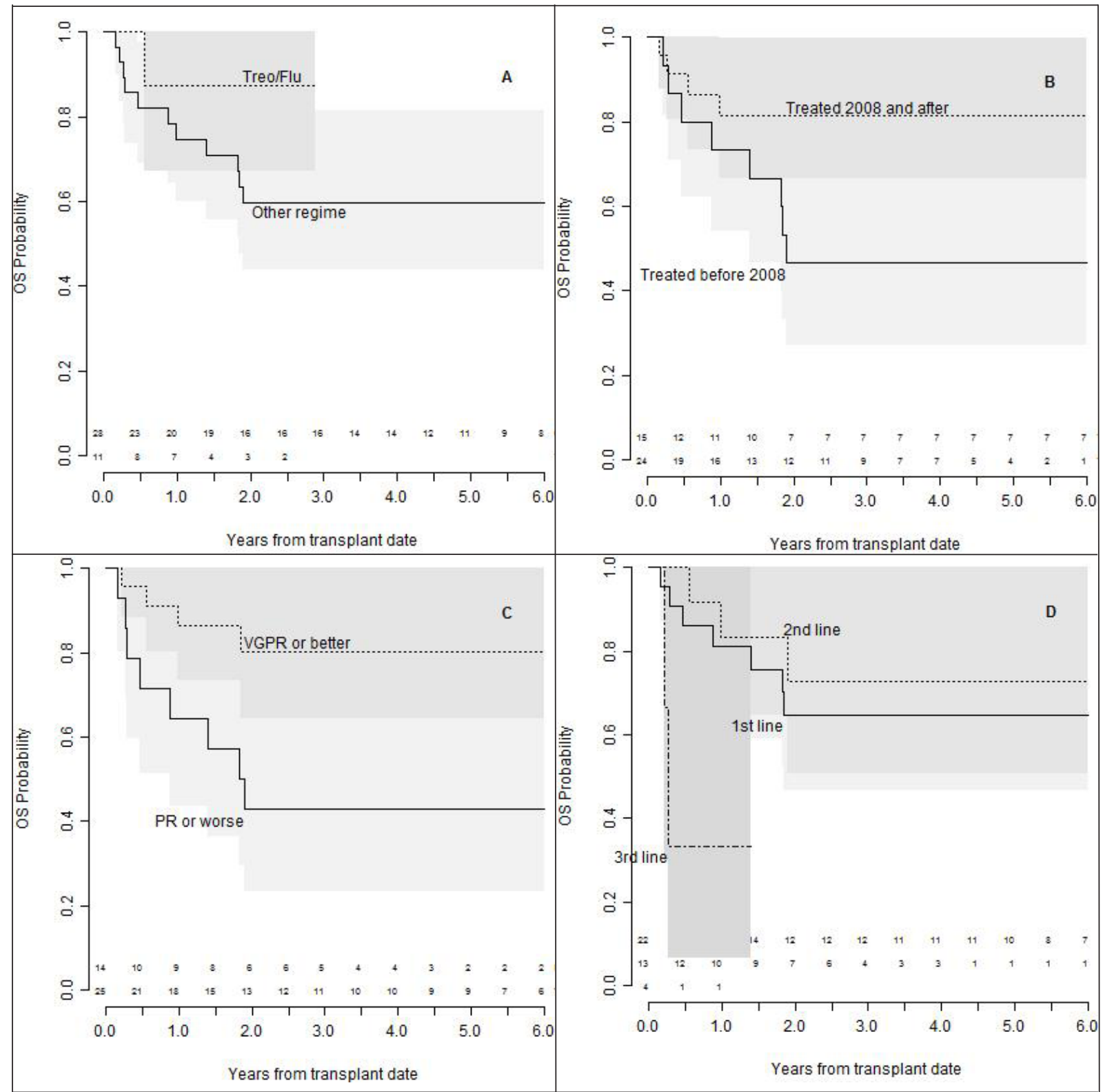

Figure 1: Overall Survival (OS), A: TreoFlu compared to all other conditioning regimen. B: Year of Allo-SCT C: Type of response before AlloSCT-SCT. D: line of treatment before Allo-SCT (lines of treatment.)

Standard induction therapy with targeted agents followed by HDT and ASCT remains standard of care for patients with multiple myeloma. For those with relapse more than 24 months after $1^{\text {st }}$ remission we most often consider a repeated HDT+ASCT. However for patients with what is considered as an early relapse ( $<18$ months) and, apparently, for this reason a more aggressive disease, there is no established standard of care.

Allo-SCT was associated with high NRM in earlier studies using myeloablative conditioning [2]. However the introduction of non-myeloablative reduced intensity conditioning (RIC) reduced NRM to $12-20 \%$ [6,7]. Although the long-term outcome appears promising in some of the studies, NRM is higher with the ASCT/RICAllo-SCT procedure compared to ASCT or ASCT/ ASCT $[8,9]$. The future of allogeneic transplantation therefore depends on improvement of NRM without hampering the GVM effect, which is important for the sustainability of response.

Although the material in this single center study is small, OS is significantly better in patients transplanted after 2008 as compared to those transplanted earlier. This improvement may depend on several factors: NRM - although not statistically better in early versus later transplants - tends to be low; there was only one death among eleven patients (9.1\%) in the small group receiving the TreoFlu combination for conditioning. Nine of the TreoFlu patients received the transplant as $2^{\text {nd }}$ or later lines of treatment. Still, the overall survival rate from the time of transplant was $89 \%$ up to 3 years from transplant with a median follow up of 1.5 years. Many changes have occurred between the early and late periods in the prevention and treatment of infections that might have impacted on the NRM. 
Our use of lenalidomide, together with DLI, after transplant from 2008 may be of importance for the outcome. Our policy has been to add lenalidomide in patients with increase of $\mathrm{M}$-component also at very low levels and in patients not entering CR after Allo-SCT (maintenance). Although lenalidomide maintenance after Allo-SCT in patients with high risk of relapse was reported to induce GVHD [10], this is not our experience in the current study, perhaps due to subsequent dose escalation of lenalidomide and later start of the maintenance treatment. However, also the TreoFlu conditioning used in half of the patients transplanted after 2008 may be of importance. This regimen may be somewhat more intensive than the previously used RIC regimens, in particularly compared to the 2 Gy TBI with or without fludarabine that was used in recent prospective studies and as well in our patients treated before 2008 .

In conclusion, we show that the outcome after Allo-SCT in multiple myeloma has improved since 2008. Treosulfan/fludarabine is a promising conditioning regimen with encouraging low NRM, which may be one reason for the improved outcome. We also show that dose-escalated lenalidomide remains a suitable relapse treatment after Allo-SCT without negatively affecting OS. Prospective trials are needed in order to confirm the retrospective results in the current study.

\section{References}

1. Moreau P (2012) Death of frontline allo-SCT in myeloma. Blood 119: 6178-9.

2. Gahrton G, Tura S, Ljungman P, Blade J, Brandt L, et al. (1995) Prognostic factors in allogeneic bone marrow transplantation for multiple myeloma. J Clin Oncol 13: 1312-22

3. Danylesko I, Shimoni A, Nagler A (2012) Treosulfan-based conditioning before hematopoietic SCT: more than a BU look-alike. Bone Marrow Transplant 47: 5-14.

4. Michallet M, Sobh M, Milpied N, Bay JO, Furst S, et al. (2012) Phase II prospective study of treosulfan-based reduced-intensity conditioning in allogeneic HSCT for hematological malignancies from 10/10 HLA-identical unrelated donor. Ann hematol 91: 1289-97.

5. Schmidt-Hieber M, Blau IW, Trenschel R, Andreesen R, Stuhler G, et al. (2007) Reduced-toxicity conditioning with fludarabine and treosulfan prior to allogeneic stem cell transplantation in multiple myeloma. Bone Marrow Transplant 39: 389-96.

6. Storb RF, Champlin R, Riddell SR, Murata M, Bryant S, et al. (2001) Non-myeloablative transplants for malignant disease. Hematology Am Soc Hematol Educ Program 375-91.

7. Gahrton G, Krishnan A (2014) Allogeneic transplantation in multiple myeloma. Expert review of hematology 7: 79-90.

8. Sahebi F, Iacobelli S, Biezen AV, Volin L, Dreger P, et al. (2015) Comparison of upfront tandem autologous-allogeneic transplantation versus reduced intensity allogeneic transplantation for multiple myeloma. Bone marrow transplant 50: 802-7.

9. Giaccone L, Storer B, Patriarca F, Rotta M, Sorasio R, et al. (2011) Long-term follow-up of a comparison of nonmyeloablative allografting with autografting for newly diagnosed myeloma. Blood 117: 6721-7.

10. Tsukada N, Shingaki S, Ikeda M, Miyazaki K, Meshitsuka S, et al. (2015) Graft-versus-host disease associated with lenalidomide maintenance after allogeneic transplantation for relapsed/refractory multiple myeloma. Rinsho Ketsueki 56: 895-900.

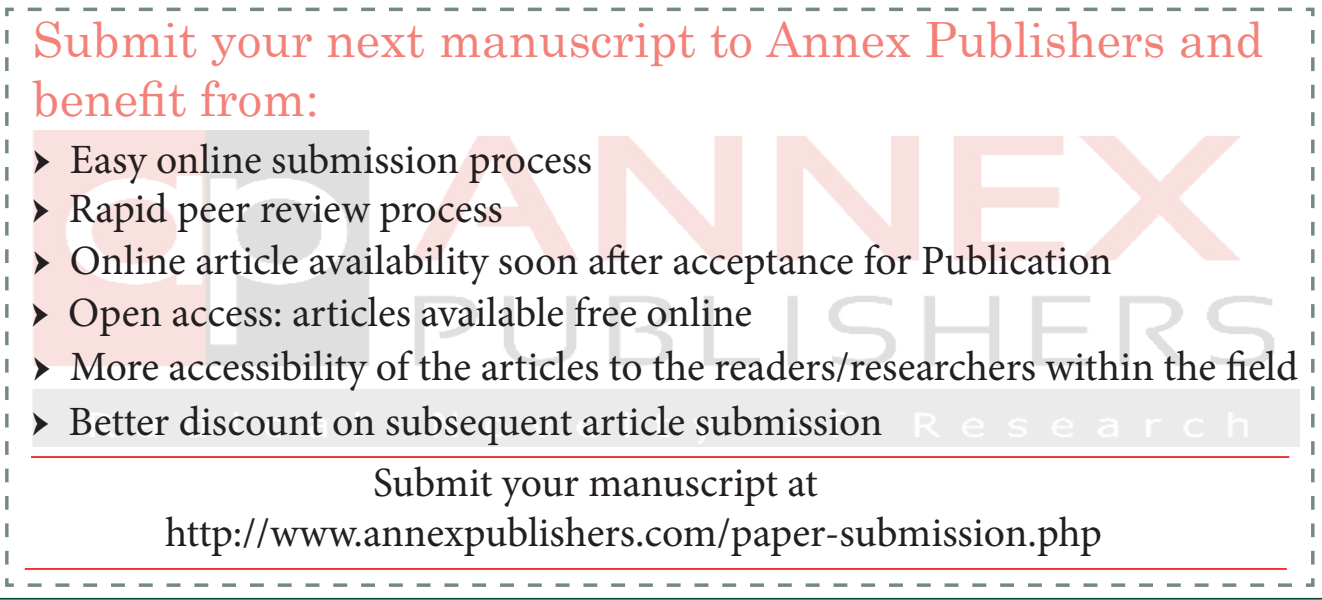

